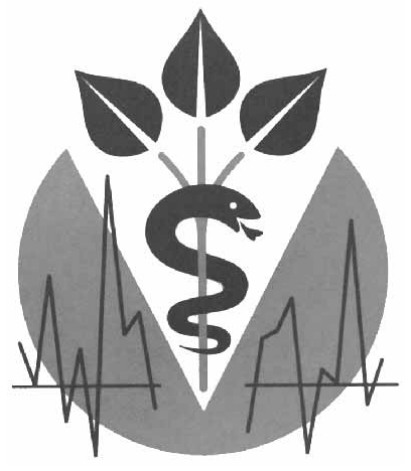

Karl und Veronica Carstens-Stiftung im Stifterverband für die Deutsche Wissenschaft Barkhovenallee 1 45239 Essen

Tel.: 02 01/84 01-111

Fax: 02 01/84 01-302

\section{Laufende Projekte}

Dr. O. Weingärtner, Bensheim:

Modellbildung und -simulation für den «arzneilichen Gehalt» homöopathischer Potenzen

Dr. P. Schirmer, Argentalklinik, Isny/Neutrauchburg, Prof. Dr. W. H. Jäckel, U Freiburg:

Klinische Studie Wirksamkeit von Formica rufa und von Eigenblutinjektionen bei Spondylitis ankylosans

Prof. Dr. H. K. Selbmann, Institut für Med. Informationsverarbeitung an der U Tübingen:

Biometrisches Zentrum für Erfahrungsmedizin (BZE) der Carstens-Stiftung

\section{Förderschwerpunkte}

- 1985-1992: Biochemische Grundlagen der Homöopathie

- 1985-1990: Wirksamkeitsnachweis von Galphimia glauca bei Heuschnupfen

- 1987-1994: Untersuchungen zu den immunmodulatorisch wirksamen Bestandteilen von Thuja occidentalis

- 1987-1997: Wirksamkeitsnachweis von Ruta graveolens bei Multipler Sklerose

- 1987-1996: Homöopathie in der Tiermedizin

- 1988-1991: Dokumentation der wissenschaftlichen Literatur zur Homöopathie

- Seit 1985: Physikalisch-chemische Untersuchungen zum Potenzierungsvorgang

- Seit 1991: Wilseder StudentInnen-Forum für Homöopathie und Essener Forum für Homöopathie

- Seit 1992: Alternative Methoden in der Diagnostik

- Seit 1993: Ambulanz für Naturheilkunde der Universitäts-Frauenklinik Heidelberg

- Seit 1994: Homöopathie in der Pädiatrie

- Seit 1997: Ambulanz für Naturheilkunde im Universitätsklinikum Freiburg (Abteilung Innere Medizin II und Institut für Umweltmedizin und Krankenhaushygiene)

- Seit 1997: Verlaufsstudie bei Patienten in der homöopathischen Arztpraxis
Prof. Dr. A. Ogilvie, Dr. G. Steidl, Institut für Biochemie, Med. Fakultät der Universität Erlangen-Nürnberg:

Zellbiologische Effekte von Ozoniden

Dr. R. Pothmann, Ev. Krankenhaus, Sozialpädiatrisches Zentrum, Oberhausen: Evaluation der Kinesiologie in der Pädiatrie

PD Dr. Karin Kraft, Med. Poliklinik der Universität Bonn:

Regulation der kapillären Durchblutung

Prof. Dr. Gisela Charlotte Fischer, Medizinische Hochschule Hannover, Abt. Allg. Medizin:

Grundlagen, Möglichkeiten und Grenzen von Naturheilverfahren und Homöopathie - ein Lehrmodell an der MHH

Prof. Dr. S. Willich, Institut für Epidemiologie, Sozial- und Präventionsmedizin, Klinikum Charité der Humboldt-Universität, Berlin:

Verlaufsstudie bei Patienten in der homöopathischen Arztpraxis

Dr. Tamara Grummt, Oelsnitz/Vogtland: Dokumentation der antikanzerogenen Wirkungen von Pflanzeninhaltsstoffen

Prof. Dr. Dr. H. E. Blum, Prof. Dr. F. D. Daschner, Institut für Umweltmedizin und Krankenhaushygiene, Freiburg: Ambulanz für Naturheilkunde am Universitätsklinikum Freiburg

PD Dr. Ingrid Kästner, Karl-Sudhoff-Institut für Geschichte der Medizin und Naturwissenschaften, Universitätsklinikum Leipzig:

Geschichte der Homöopathie in Russland

Münchener Modell, TU und LMU München:

Übersichtsarbeiten zur Komplementärmedizin im Rahmen der Cochrane Collaboration

Dr. K. Linde, Münchener Modell, TU und LMU München:

Förderpreis 1997 der Carstens-Stiftung 


\section{Promotionsstipendien}

Y.Jin, Ambulanz für Naturheilkunde der Universitätsfrauenklinik Heidelberg:

Anwendung der Traditionellen Chinesischen Medizin

\section{H. Brockmeyer, Giessen:}

Zum Einfluss der Ohrakupunktur bei einer psychischen Belastungssituation

P. Lehrke, Freiburg:

Impfverhalten bei homöopathischen Ärzten

\section{Lill, München:}

Schmerzlindernde Wirkung von Akupunktur bei Halsschmerzen

\section{Schellenberg, Giessen:}

Beeinflussung des Schluck- und Würgereflexes durch Akupunktur

\section{J. Alex, Tübingen:}

Homöopathisches Thyroxin und Metamorphose

\section{W. Doerfler, Heidelberg:}

Behandlung des klimakterischen Symptomenkomplexes mit der Traditionellen Chinesischen Medizin (TCM)

U. Albrecht, Woltersdorf:

Schröpfbehandlung der Brachialgia parästhetica nocturna

\section{Zimmer, Saarbrücken:}

Die saarländische und chinesische Maulwurfsgrille in der Wundheilkunde

\section{Neue Bewilligung}

Immunmodulierende und Photosensibilisierende Wirkungen von Johanniskraut-Creme

\section{Summe: $17000,-$ DM}

Ansprechpartner: Dr. Christoph M. Schempp, Universitäts-Hautklinik Freiburg

Das Johanniskraut, Hypericum perforatum $L$., ist auf der ganzen Welt verbreitet und zählt zu den klassischen Phytopharmaka. Sein Gebrauch geht bis in die Antike zurück. Traditionell werden ölige Extrakte des Johanniskrautes zur Behandlung von Wunden, Verbrennungen und Nervenverletzungen verwendet. In der Homöopathie wird das Johanniskraut bei Störungen des ZNS, bei funktionellen Depressionen und bei ekzemartigen Hautausschlägen angewendet.

In neueren, zum Teil placebokontrollierten Studien wurde die antidepressive Wirkung eines standardisierten Hypericum-Extraktes nachgewiesen. Die Wirkung war derjenigen trizyklischen Antidepressiva vergleichbar, ohne dass es zum Auftreten unerwünschter Arzneimittelwirkungen kam. Als mögliche Grundlage der antidepressiven Wirkung von Johanniskraut-Extrakt wird die Hemmung der Monoamino-Oxidase (MAO) durch Hypericin angesehen. Diese Wirkung ist unabhängig von der Aktivierung des Hypericins durch sichtbares Licht. Neuere Untersuchungen haben ergeben, dass Hypericin auch eine lichtabhängige, antiretrovirale Aktivität besitzt. Es ist zu erwarten, dass eine Reihe weiterer lichtabhängiger Wirkungen von Hypericin existieren. Das Auftreten von ekzemartigen Ausschlägen bei der homöopathischen Arzneimittelprüfung und die Beobachtung von phototoxischen Reaktionen an der Haut von Weidetieren lassen eine Beziehung des Johanniskrautes zum Hautorgan erkennen.

Kann Johanniskraut-Extrakt auch bei der Behandlung von Hautkrankheiten genutzt werden?

Hierüber gibt es bisher keine wissenschaftlichen Untersuchungen. Vor einer klinischen Anwendung muss jedoch zuerst geprüft werden, ob z. B. Johanniskraut-Creme gut verträglich ist, denn «die Dosis macht das Gift» (Paracelsus). Insbesondere muss ausgeschlossen werden, dass es wie bei Weidetieren zu einer starken Erhöhung der Lichtempfindlichkeit kommt. Nur dann können die heilenden Wirkungen der Johanniskraut-Creme bei der Behandlung von Hautkrankheiten wie Neurodermitis oder Schuppenflechte genutzt werden.

Deshalb führt die Universitäts-Hautklinik Freiburg mit Unterstützung der Karl und Veronica Carstens-Stiftung eine Pilotstudie durch, bei der getestet werden soll, ob es beim Menschen

1. zu einer Erhöhung der Lichtempfindlichkeit und

2. zu einer therapeutisch nutzbaren immunologischen Beeinflussung der Haut kommt.

Beide Effekte könnten therapeutisch zur Behandlung von Hautkrankheiten genutzt werden. Vor einer therapeutischen Anwendung muss jedoch eine starke Phototoxizität der Johanniskraut-Creme ausgeschlossen werden. Mit der geplanten Studie wird ein erster Schritt zur Erforschung der seit langem praktizierten lokalen Anwendung von Johanniskraut-Extrakt getan. 